\title{
Comparison of the amino acid and peptide composition and postprandial response of beef, hydrolyzed chicken, and whey protein nutritional preparations
}

\author{
Christopher J. Detzel, Bryon W. Petschow, Nicole Johnson, and Eric M. Weaver
}

Essentia Protein Solutions, 2527 SE Oak Tree Ct, Ankeny, IA 50021, USA

Corresponding Author: Christopher Detzel, PhD, Essentia Protein Solutions, 2527 SE Oak Tree Ct, Ankeny, IA 50021, USA

Submission Date: October 3, 16, 2016, Accepted Date: October 25, 2016, Publication Date: October 30, 2016

Citation: Detzel C.J, Petschow B.W., Johnson N., and Weaver E.M.. Comparison of the amino acid and peptide composition and postprandial response of beef, hydrolyzed chicken, and whey protein nutritional preparations. Functional Foods in Health and Disease 2016; 6(10): 612-626

\begin{abstract}
Background: Increasing dietary protein intake synergistically improves the effect of exercise to stimulate muscle protein synthesis. The purpose of this study was to evaluate the plasma amino acid response of two novel protein nutritional preparations, beef protein isolate (BeefISO ${ }^{\mathrm{TM}}$ ) and hydrolyzed chicken protein isolate $\left(\mathrm{MyoCHX}^{\mathrm{TM}}\right)$.
\end{abstract}

Methods: The postprandial plasma amino acid response over 3 hours was monitored in young adults $(n=6)$ following consumption of 23 grams of WPC, BeefISO ${ }^{\mathrm{TM}}$, or MyoCHX ${ }^{\mathrm{TM}}$. Amino acid compositional analysis and molecular weight distributions of each protein were performed by HPLC. Statistical analyses were performed using one-way or two-way ANOVA where appropriate and corrected for multiple comparisons to account for the cross-over design.

Results: Compositional evaluations revealed similar levels of essential and branched-chain amino acids for WPC and MyoCHX ${ }^{\mathrm{TM}}$. While the results of this study predictably demonstrated plasma amino acids levels increased following consumption of the different proteins, the kinetics of the postprandial response was unique to each protein source. WPC and MyoCHX ${ }^{\mathrm{TM}}$ were rapidly absorbed with maximum plasma amino acid concentrations observed at 30 and 15 min, respectively. The slightly faster absorption of $\mathrm{MyoCHX}{ }^{\mathrm{TM}}$ was associated with the increased peptide content of MyoCHX ${ }^{\mathrm{TM}}$ (greater than $76 \%$ of protein is $<2 \mathrm{kDa}$ ). BeefISO ${ }^{\mathrm{TM}}$ exhibited sustained release characteristics as evidenced by increased post prandial amino acid concentrations after 3 hours.

Conclusions: The protein preparations studied each had different amino acid profiles and 
absorption kinetics. WPC and MyoCHX ${ }^{\mathrm{TM}}$ contained a higher essential amino acid content and were rapidly absorbed with plasma amino acid concentrations peaking within 30 minutes following consumption. BeefISO ${ }^{\mathrm{TM}}$ contained a higher proportion of conditionally essential amino acids that steadily increased in plasma over 3 hours, indicating a sustained release profile for BeefISO ${ }^{\mathrm{TM}}$. Each of these three protein preparations have been shown to facilitate similar improvements in body composition following a resistance-exercise training program. The paradoxical finding that proteins with varying composition, molecular weight, and digestibility can lead to similar effects on body composition suggests the need to evaluate proteins based on total nutritional potential rather than as a delivery vehicle for single amino acids.

Key Words: Protein digestibility; amino acids; body composition; beef protein; chicken protein

\section{BACKGROUND}

Numerous studies have shown that increasing dietary protein intake has a synergistic effect on the impact of resistance exercise to stimulate muscle protein synthesis (MPS) [1]. The increase in MPS that follows resistance exercise and protein feeding occurs in both young [1-4] and older adults [5, 6]. Ingestion of as little as 10 grams of protein after resistance exercise has been shown to stimulate higher rates of MPS compared to exercise alone [3,7]. This increase in MPS is most often attributed to the postprandial rise in circulating essential amino acids (EAAs) that follows ingestion of a protein meal [8]. Three EAAs known as the Branched Chain Amino Acids (BCAA; leucine, isoleucine and valine) are believed to play particularly important roles in stimulating MPS, since they make up a high proportion of the amino acids (AAs) found in muscle. Leucine is the most abundant BCAA found in muscles and is recognized as a potent stimulator of MPS through its actions on intracellular regulatory proteins involved in the rapamycin (mTOR) signaling cascade [9, 10]. However, a full complement of EAAs may also be an important consideration for sustaining exercise-induced rates of MPS [11].

It is generally accepted that athletes and adults involved in resistance training have a higher dietary protein requirement compared to less active individuals due to the additional protein/amino acids needed to repair and rebuild skeletal muscle following intense training bouts or athletic events. Accordingly, supplementing the diet with additional protein has become very popular among athletes, both amateur and professional, who want to build muscle tissue and speed up recovery after rigorous training sessions. Not surprisingly, this has led to an increase in the different types of proteins that are available to athletes or other adults who wish to build or retain muscle tissue and speed up recovery after rigorous training sessions. While the quality of the protein is most frequently tied to the levels of EAAs, studies have also shown that the source of protein $[4,12,13]$ can be an important factor in determining the extent of MPS following protein ingestion. For example, the overall digestibility of the protein source as well as other non-protein components that may be present can affect the impact of protein sources on MPS. Meat is one such example since it can be an important source of various micronutrients, such as creatine, zinc, B vitamins, iron, selenium, potassium, and other vitamins and minerals $[3,14,15]$.

Much of the research to date has been focused on animal and dairy-based products because they contain the highest percentage of EAAs and result in greater protein synthesis and muscle hypertrophy following resistance training compared to a vegetarian protein-matched control, 
which typically lack one or more EAAs $[3,16,17]$. However, few studies have evaluated the postprandial rise in amino acids as an indicator of protein digestibility for animal and dairy protein sources. The purpose of this study was to evaluate plasma amino acid profiles following consumption of whey protein concentrate (WPC), beef protein isolate $\left(\mathrm{BeefISO}^{\mathrm{TM}}\right.$ ), or hydrolyzed chicken protein isolate (MyoCHX ${ }^{\mathrm{TM}}$ ), in healthy, young adult subjects. Amino acid absorption was studied using a cross-over design with a 6-day washout period between protein supplements with postprandial amino acid levels monitored for 3 hours following ingestion of each protein source.

\section{METHODS}

\section{Study Population}

Six normal healthy non-pregnant subjects ( 3 males, 3 females) between 20-28 years of age were recruited from the campus at the University of Tampa and considered eligible for the study. Subjects were screened to ensure that they met and would adhere to the following criteria prior to entry into the study: (1) maintain a diet consisting of $15-20 \%$ protein, $45-55 \%$ carbohydrate, and 25-30\% fat; (2) not taking performance enhancing supplements for the previous 6 weeks; (3) non-smokers; (4) not taking amino acid supplements; (5) not using anabolic or catabolic hormones; and (6) not on medication or supplements known to influence any of the variables measured in the study. Written informed consent was obtained from all study participants, and the protocol was approved by the University of Tampa Institutional Review Board.

\section{Study Design}

The cross-over study ( $\mathrm{n}=6$ ) involved consumption of protein supplements one day per week for 3 weeks, with a 6-day washout period between protein supplementations, to evaluate plasma amino acid profiles following ingestion of protein supplement shakes made from WPC, BeefISO ${ }^{\mathrm{TM}}$, or $\mathrm{MyoCHX}^{\mathrm{TM}}$. The proteins supplements were prepared with basal protein powders and a flavoring system to provide 23 grams of protein. The basal protein powders were WPC (Agropour Ingredients, La Crosse, WI), BeefISO ${ }^{\text {TM }}$ (Essentia Protein Solutions, Ankeny, IA) and MyoCHX $^{\text {TM }}$ (Essentia Protein Solutions). Six subjects were fasted overnight ( $\geq 8$ hours) and then consumed a protein shake containing 23 grams of one of the test protein sources. Subjects were not allowed to consume water 1 hour before or 1 hour after consumption of investigational product. Subjects fasted overnight and food was not allowed for at least 3 hours following ingestion of study product. Serial blood samples were collected at time 0 (immediately prior to ingestion of the protein) and at 15,30, 45, 60, 90, 120 and 180 minutes after consuming the study product. This process of overnight fasting, consumption of test shake, and sequential blood draws was repeated weekly for three weeks to evaluate each of the different protein sources.

\section{Evaluation of protein molecular weight distribution}

Peptide molecular weight (MW) distribution for the various protein sources (WPC, BeefISO ${ }^{\mathrm{TM}}$, and $\mathrm{MyoCHX}^{\mathrm{TM}}$ ) was estimated by size exclusion High Performance Liquid Chromatography (HPLC) using a method similar to that described by Karamać and Rybarczyk [18]. The HPLC system consisted of a Waters 1525 Binary Pump, Waters 2487 dual absorbance Detector, and Waters 717 Plus Auto sampler; the system utilized Empower Pro 5.0 software (Waters). A TOSOH TSKgel G2000SW $\mathrm{xl}_{\mathrm{xl}}(125 \AA, 5 \mu \mathrm{m}, 300 \mathrm{x} 7.8 \mathrm{~mm})$ size exclusion column with matching 
guard column $(40 \times 6.0 \mathrm{~mm})$ was used to separate proteins 1-29 kilodaltons (kDa) in size. GraphPad Prism 6.0 (GraphPad Software Inc., La Jolla, CA) was used to graph the sigmoidal relationship between retention time and log molecular weight for the standard curve. Standards were a mix of Albumin from bovine serum, Carbonic Anhydrase from bovine erythrocytes, Cytochrome $\mathrm{C}$ from equine heart, Insulin Chain B oxidized from bovine pancreas, Aprotinin from bovine lung, and Bradykinin Acetate Salt. HPLC mobile phases and sample prep buffers were made from HPLC grade Acetonitrile (ACN), HPLC grade water, and Trifluoroacetic Acid (TFA; Thermo Fisher Scientific, Waltham, MA); all HPLC standards and reagents were purchased from Sigma-Aldrich (St. Louis, MO) unless otherwise noted.

Protein samples were dissolved in water to make $10 \%(\mathrm{w} / \mathrm{w})$ stock and diluted to $1 \%(\mathrm{v} / \mathrm{v})$ using $45 \%$ ACN. Standards were similarly prepared in $45 \%$ ACN. Duplicate $10 \mu$ injections of standard or sample were separated under isocratic flow of $0.63 \mathrm{~mL} / \mathrm{min}$ of $71.6 \%$ ACN with $0.1 \%$ TFA and $0.37 \mathrm{~mL} / \mathrm{min}$ of HPLC grade water with $0.1 \%$ TFA. Protein absorbance chromatograms $(215 \mathrm{~nm})$ were analyzed using Empower Pro 5.0 software for peak retention times which were compared with the standard curve to determine protein/peptide molecular weight distribution of the different protein sources.

\section{Evaluation of plasma amino acid profiles}

Plasma amino acid analysis was conducted by liquid chromatography-electrospray-tandem mass spectrometry (LC/ES/MS) using an Agilent 1200/6460 triple quadrupole LC/MS system (Santa Clara, CA) by Heartland Assays (Ames, IA). Standards and $25 \mu$ l of plasma were processed using a EZ:faastTM (Phenomenex, Torrance, CA) analysis kit for physiological amino acid by LC-MS [19]. Amino acids that were quantified included all of those found in the test proteins (Table 1). Plasma amino acid concentration-time curves for each subject and protein source were prepared by calculating the difference between measured amino acid levels at individual time points and the average of the baseline values $(t=0)$ measured at each of the three crossover periods.

\section{Statistical analysis}

Amino acid response curves were evaluated using a two-way ANOVA with repeated measures to account for the serial plasma collections over 3 hours. Multiple comparisons within protein groups were corrected using the Dunnett method to evaluate changes in amino acid concentration from baseline. Baseline amino acid concentration was determined for each subject using the average of the baseline $(\mathrm{t}=0)$ amino acid concentration across all proteins in the cross-over study. Multiple comparisons between proteins were corrected using Tukey's adjustment for multiple comparisons. Tests for significant amino acid responses resulting in an increase from baseline and test comparing the response between proteins at a single time-point were conducted using unadjusted plasma amino acid concentrations. However, area under the curve measurements were calculated based on postprandial plasma amino acid concentrations being adjusted to the average baseline discussed above. Area under the curve measurements were calculated and statistical comparisons were made using a One-way ANOVA which was corrected for multiple comparisons using Tukey's adjustment. Statistical significance was set at $\mathrm{p}<0.05$ unless otherwise noted. 


\section{RESULTS}

\section{Composition of Test Protein Sources}

The amino acid compositions of WPC, BeefISO ${ }^{\mathrm{TM}}$, MyoCHX ${ }^{\mathrm{TM}}$ are shown in Table 1. The $\mathrm{MyoCHX}^{\mathrm{TM}}$ and BeefISO ${ }^{\mathrm{TM}}$ protein sources each contain over 90\% protein (grams/100 grams dry weight), while the WPC source was $80 \%$ protein. However, in this study the amino acid content of each protein source was normalized to provide 23 grams of protein. As expected, there was considerable variation in the amino acid composition of the WPC, BeefISO ${ }^{\mathrm{TM}}$, MyoCHX ${ }^{\mathrm{TM}}$ protein sources. The essential amino acid content was similar for WPC (37\%) and MyoCHX ${ }^{\mathrm{TM}}$ $(35 \%)$, but lower for BeefISO ${ }^{\mathrm{TM}}(18 \%)$. Likewise, BCAA content for WPC, MyoCHX ${ }^{\mathrm{TM}}$ and BeefISO $^{\text {TM }}$ was approximately $18 \%, 15 \%$, and $8 \%$, respectively. The greatest differences in individual amino acid levels (e.g. 3-fold or greater for any 2 of the protein sources) were observed for arginine, cysteine, glycine, isoleucine, tryptophan, and hydroxyproline, Table 1.

Table 1. Amino acid composition of protein supplement sources

\begin{tabular}{|c|c|c|c|}
\hline Protein Component & WPC & BeefISO $^{\text {TM }}$ & МyоСНХТм \\
\hline Total Protein ( $\%$ of dry material) & 80.0 & 98.3 & 91.2 \\
\hline $\begin{array}{l}\text { EAAs }(\mathrm{gm} / 100 \text { gm protein }) \\
\text { BCAAs }(\mathrm{gm} / 100 \text { gm protein }) \\
\text { CEAAs }(\mathrm{gm} / 100 \text { gm protein })\end{array}$ & $\begin{array}{l}37.3 \\
17.7 \\
29.5\end{array}$ & $\begin{array}{c}18.1 \\
8.0 \\
51.1\end{array}$ & $\begin{array}{l}34.8 \\
14.7 \\
36.8\end{array}$ \\
\hline \multicolumn{4}{|l|}{${\text { Individual Amino Acids }{ }^{1}}^{2}$} \\
\hline Alanine & 3.8 & 8.1 & 5.7 \\
\hline Arginine & 1.9 & 6.9 & 5.6 \\
\hline Aspartic acid & 8.7 & 5.8 & 8.1 \\
\hline Cysteine & 1.4 & 0.1 & 0.8 \\
\hline Glutamic acid $\dagger$ & 13.7 & 10.5 & 13.2 \\
\hline Glycine & 1.6 & 19.4 & 6.3 \\
\hline Histidine & 1.4 & 1.1 & 2.6 \\
\hline Isoleucine & 5.0 & 1.6 & 3.8 \\
\hline Leucine & 8.2 & 3.6 & 6.6 \\
\hline Lysine & 7.1 & 3.6 & 7.1 \\
\hline Methionine & 1.6 & 0.9 & 2.2 \\
\hline Phenylalanine & 2.6 & 2.2 & 3.2 \\
\hline Proline & 4.8 & 10.1 & 4.6 \\
\hline Serine & 4.0 & 3.1 & 3.6 \\
\hline Threonine & 5.2 & 2.1 & 4.2 \\
\hline Tryptophan & 1.6 & 0.2 & 0.9 \\
\hline Tyrosine & 2.1 & 1.0 & 2.7 \\
\hline Valine & 4.6 & 2.8 & 4.2 \\
\hline Hydroxyproline & - & 9.7 & 1.5 \\
\hline
\end{tabular}

${ }^{1}$ Data expressed as gram (gm) of amino acid per $100 \mathrm{gm}$ of protein raw material used to prepare protein supplement shakes.

$\uparrow$ Values includes both glutamic acid and glutamine due to analytical reasons. 
Evaluation of the peptide composition and levels of free amino acids also revealed considerable variation among the three protein sources (Figure 1). For example, nearly $85 \%$ of the WPC protein was composed of peptides ranging from 5-15 kDa in length, while $76 \%$ of the MyoCHX ${ }^{\mathrm{TM}}$ proteins were composed of free amino acids or peptides smaller than $2 \mathrm{kDa}$, indicating the majority of the protein being comprised of peptides shorter than 15 amino acids in length. Evaluation of the BeefISO ${ }^{\mathrm{TM}}$ material demonstrated a peptide distribution closer to the peptide composition of WPC than MyoCHX ${ }^{\mathrm{TM}}$ with approximately $68 \%$ of the protein in BeefISO $^{\mathrm{TM}}$ ranging between 5 and $15 \mathrm{kDa}$ and about $14 \%$ having a molecular weight less than 2 $\mathrm{kDa}$.

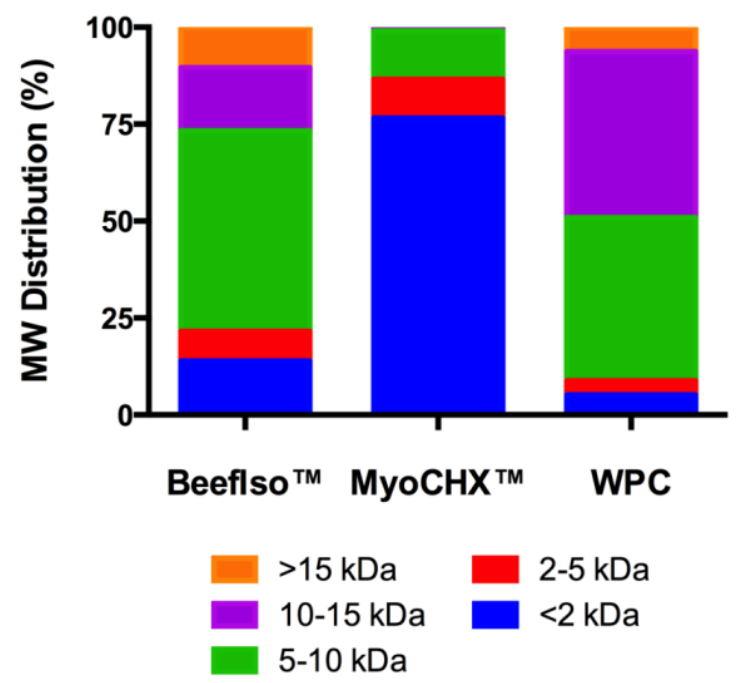

Figure 1. Peptide and free amino acid composition of protein sources. The protein/peptide molecular weight distribution as determined by HPLC is shown for whey (WPC), beef protein $\left(\right.$ BeefISO $\left.^{\mathrm{TM}}\right)$, and hydrolyzed chicken protein (MyoCHX $\left.{ }^{\mathrm{TM}}\right)$. The majority of protein/peptides are in the $5-15 \mathrm{kDa}$ range for both BeefISO ${ }^{\mathrm{TM}}$ and $\mathrm{WPC}, 68 \%$ and $85 \%$ respectively. In contrast, MyoCHX ${ }^{\mathrm{TM}}$ is composed primarily of free amino acids and peptides with $76 \%$ of the protein having a molecular weight of $<2 \mathrm{kDa}$.

\section{Plasma Amino Acid Profile after Ingestion of Test Proteins}

The postprandial digestion and absorption of protein in test supplements was assessed following an overnight fast by measuring plasma concentrations of amino acids after ingestion of a single dose (23 grams) of WPC, BeefISO ${ }^{\mathrm{TM}}$, MyoCHX ${ }^{\mathrm{TM}}$. The corresponding time course for the appearance of total amino acids (AA), essential amino acids (EAA), branched chain amino acids (BCAA), and conditionally essential amino acids (CEAA) in plasma are shown in Figure 2. Increases in plasma concentrations of total AAs, EAAs, BCAAs, and CEAAs were observed for all protein supplement groups, with peak plasma concentrations ranging from 15-60 min after consumption of the protein supplements. Plasma levels of total AAs in the MyoCHX ${ }^{\mathrm{TM}}$ group were significantly higher $(\mathrm{p} \leq 0.05)$ than subjects receiving BeefISO ${ }^{\mathrm{TM}}$ at 15 and 30 minutes and significantly increased when compared to subjects receiving WPC at $120 \mathrm{~min}$ (Figure 2A). Significantly higher $(\mathrm{p} \leq 0.05)$ levels of plasma EAAs were found in MyoCHX ${ }^{\mathrm{TM}}$ subjects when compared to BeefISO ${ }^{\mathrm{TM}}$ subjects at 15, 30, 45, 60, and $120 \mathrm{~min}$, and to WPC subjects at 15 and 
120 min (2B). Subjects receiving WPC also had significantly higher $(\mathrm{p} \leq 0.05)$ levels of plasma EAAs compared to BeefISO ${ }^{\text {TM }}$ subjects at 45 and $60 \mathrm{~min}$. Similarly, plasma levels of BCAAs were significantly higher in the MyoCHX ${ }^{\mathrm{TM}}$ group $(\mathrm{p} \leq 0.05)$ when compared to BeefISO ${ }^{\mathrm{TM}}$ subjects at 15, 30, and $45 \mathrm{~min}$, and WPC subjects at 15 and $120 \mathrm{~min}$; BCAA levels were also higher in the WPC group compared to the BeefISO ${ }^{\mathrm{TM}}$ group at 45 and 60 min (2C). In contrast, levels of plasma CEAAs in subjects consuming BeefISO ${ }^{\text {TM }}$ were higher $(\mathrm{p} \leq 0.05)$ and sustained longer when compared to the WPC group at 90, 120, and 180 min, and the MyoCHX ${ }^{\mathrm{TM}}$ group at $180 \min (2 \mathrm{D})$.
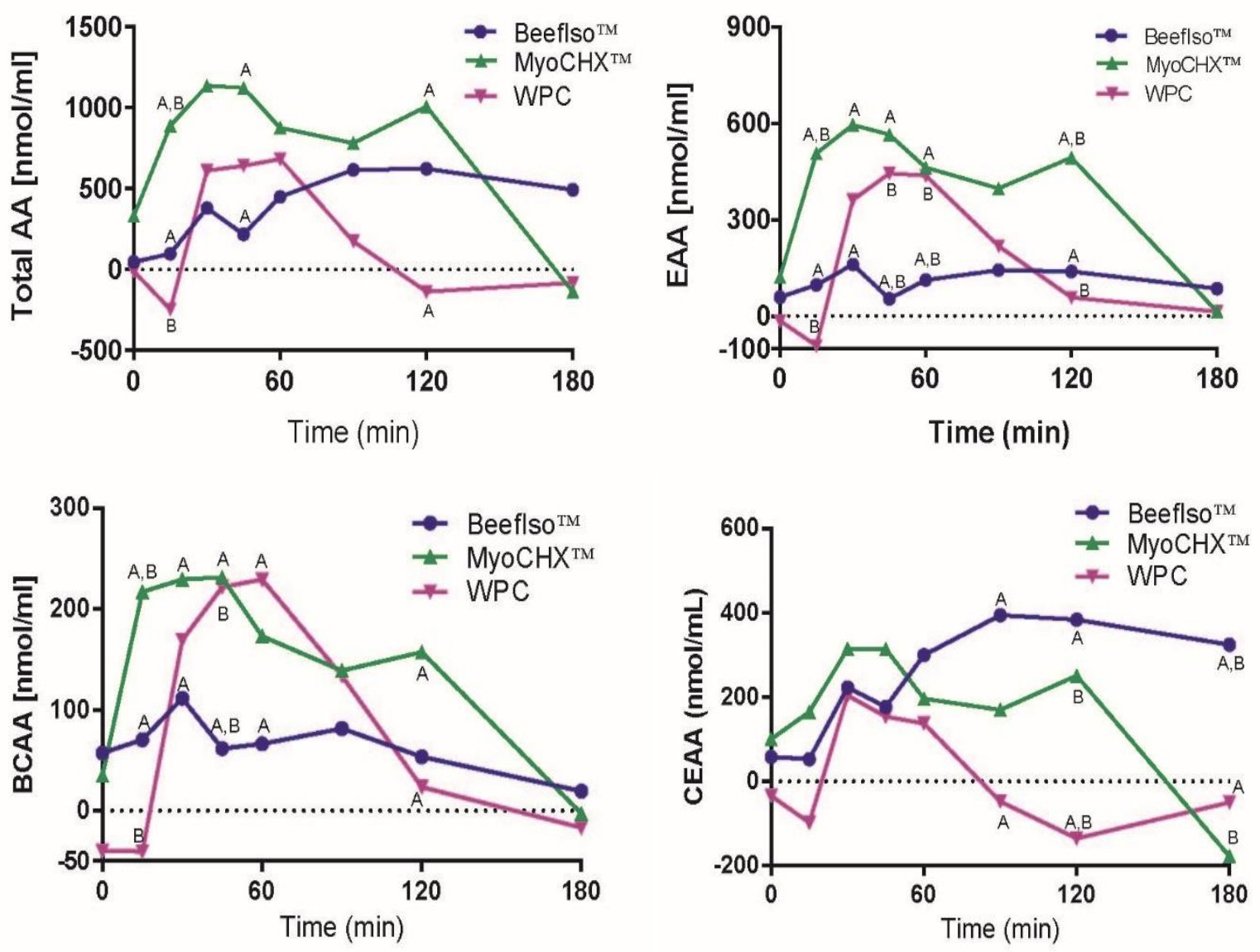

Figure 2. Plasma concentrations of amino acids. Mean plasma concentrations of total amino acids (panel A), essential amino acids (panel B), branched chain amino acids (panel C), and conditionally essential amino acids (panel D) following ingestion of WPC, BeefISO ${ }^{\mathrm{TM}}$, and MyoCHX ${ }^{\mathrm{TM}}$. Proteins sharing a common letter at a particular time point were found to have statistically different $(\mathrm{P} \leq 0.05)$ postprandial plasma amino acid responses.

As shown in Figure 3, the calculated area under the curve $\left(\mathrm{AUC}_{0-180}\right)$ for total amino acids (Figure 3A), EAA (3B), and BCAA (3C) following overnight fasting in the MyoCHX ${ }^{\mathrm{TM}}$ group tended to be higher than the calculated $\mathrm{AUC}_{0-180}$ for subjects receiving WPC or BeefISO ${ }^{\mathrm{TM}}$. There were no between-group significant differences in calculated $\mathrm{AUC}_{0-180}$ for total AAs, EAAs, or BCAA. In contrast, the calculated $\mathrm{AUC}_{0-180}$ for CEAA was statistically higher $(\mathrm{p}=0.047)$ in subjects receiving BeefISO ${ }^{\mathrm{TM}}$ compared to WPC (3D). 

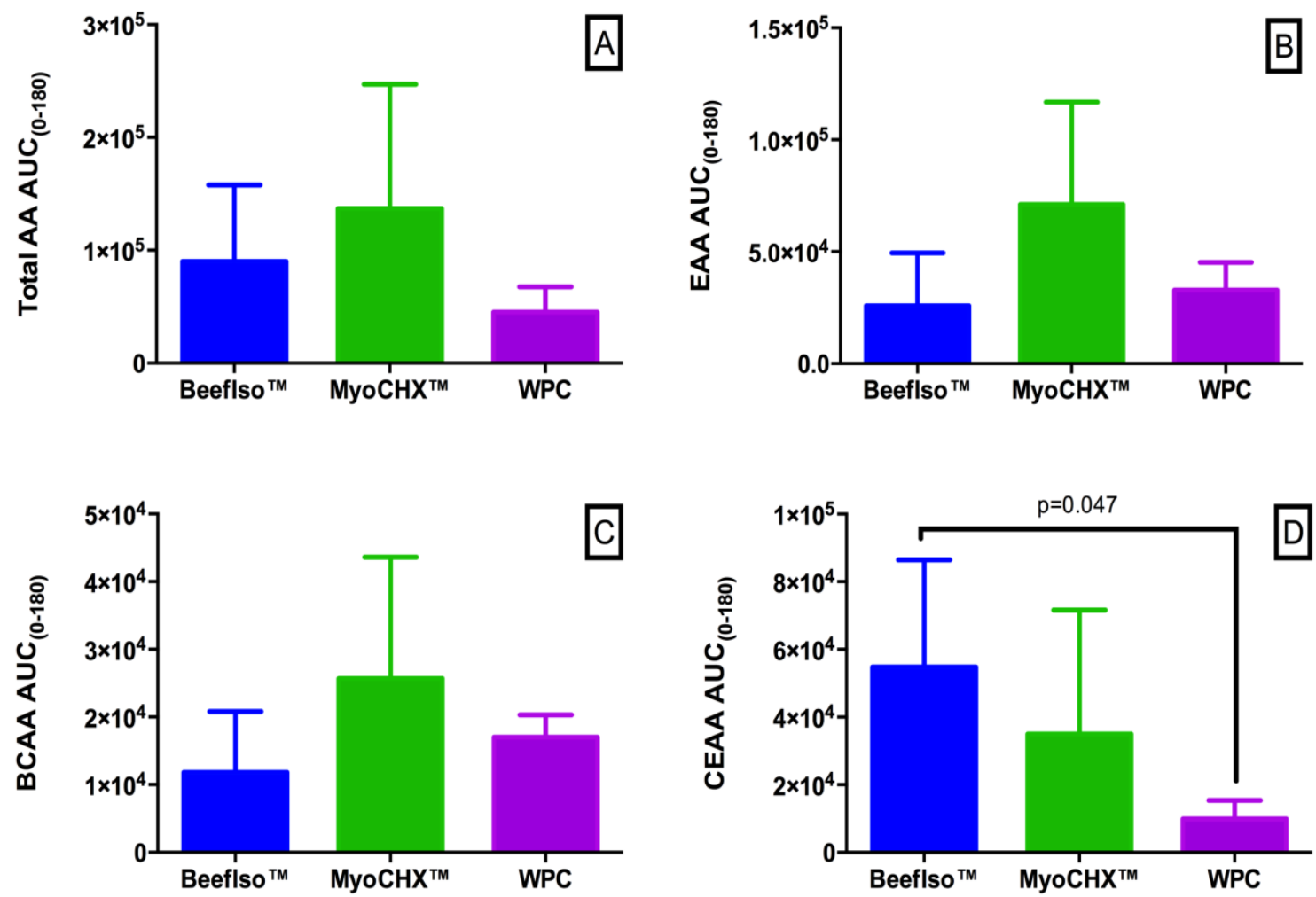

Figure 3. Mean area under the curve $\left(\mathrm{AUC}_{0-180}\right)$ for plasma amino acids. Mean area under the curve $\left(\mathrm{AUC}_{0-180}\right)$ for plasma amino acids. Area under the plasma amino acid concentration-time curve (nmol/mL*min) from time 0 to 180 minutes (AUC ${ }_{0-180}$ ) following ingestion of WPC, BeefISO $^{\mathrm{TM}}$, and $\mathrm{MyoCHX}{ }^{\mathrm{TM}}$. $\mathrm{AUC}_{0-180}$ of total amino acid response (panel A), essential amino acid response (panel B), branched chain amino acid response (panel C), and conditionally essential amino acid response (panel D) are shown. Statistical significance between protein groups was tested at $\mathrm{P}<0.05$ and is indicated on charts by linked protein treatments.

The postprandial plasma amino acid responses for select individual amino acids including leucine, arginine, glycine, and valine are shown in Figure 4. Individual amino acid responses reflected the amino acid composition of the proteins. Significantly higher $(p \leq 0.05)$ levels of leucine were found in MyoCHX ${ }^{\mathrm{TM}}$ subjects when compared to BeefISO ${ }^{\mathrm{TM}}$ subjects at $15,30,45$, 60 , and $90 \mathrm{~min}$ (4A). While WPC led to a significantly higher leucine response compared to BeefISO $^{\mathrm{TM}}$ at 30, 45, 60, and 90 min which was significantly higher than MyoCHX ${ }^{\mathrm{TM}}$ at 45, 60, and 90 min (4A). The greatest arginine response was observed for MyoCHX ${ }^{\mathrm{TM}}$ which resulted in a significantly higher plasma arginine concentration at 15, 30, and 45 min when compared to WPC, and at $15 \mathrm{~min}$ when compared to BeefISO ${ }^{\mathrm{TM}}$ (4B). Similarly, BeefISO ${ }^{\mathrm{TM}}$ led to a significantly higher plasma arginine concentration than WPC at $15 \mathrm{~min}$ and a significantly higher plasma arginine concentration than $\mathrm{MyoCHX}^{\mathrm{TM}}$ at 180 min. BeefISO ${ }^{\mathrm{TM}}$, being rich in glycine, led to significantly increased plasma glycine concentrations at 60, 90, and 120 when compared to WPC and significantly higher glycine concentration than MyoCHX ${ }^{\mathrm{TM}}$ at 60 and 180 min (4C). Finally, postprandial plasma valine concentrations are shown in Figure 4D which demonstrates a varied response between the three proteins, with MyoCHX ${ }^{\mathrm{TM}}$ being significantly higher than WPC at 15 and 120 min. 

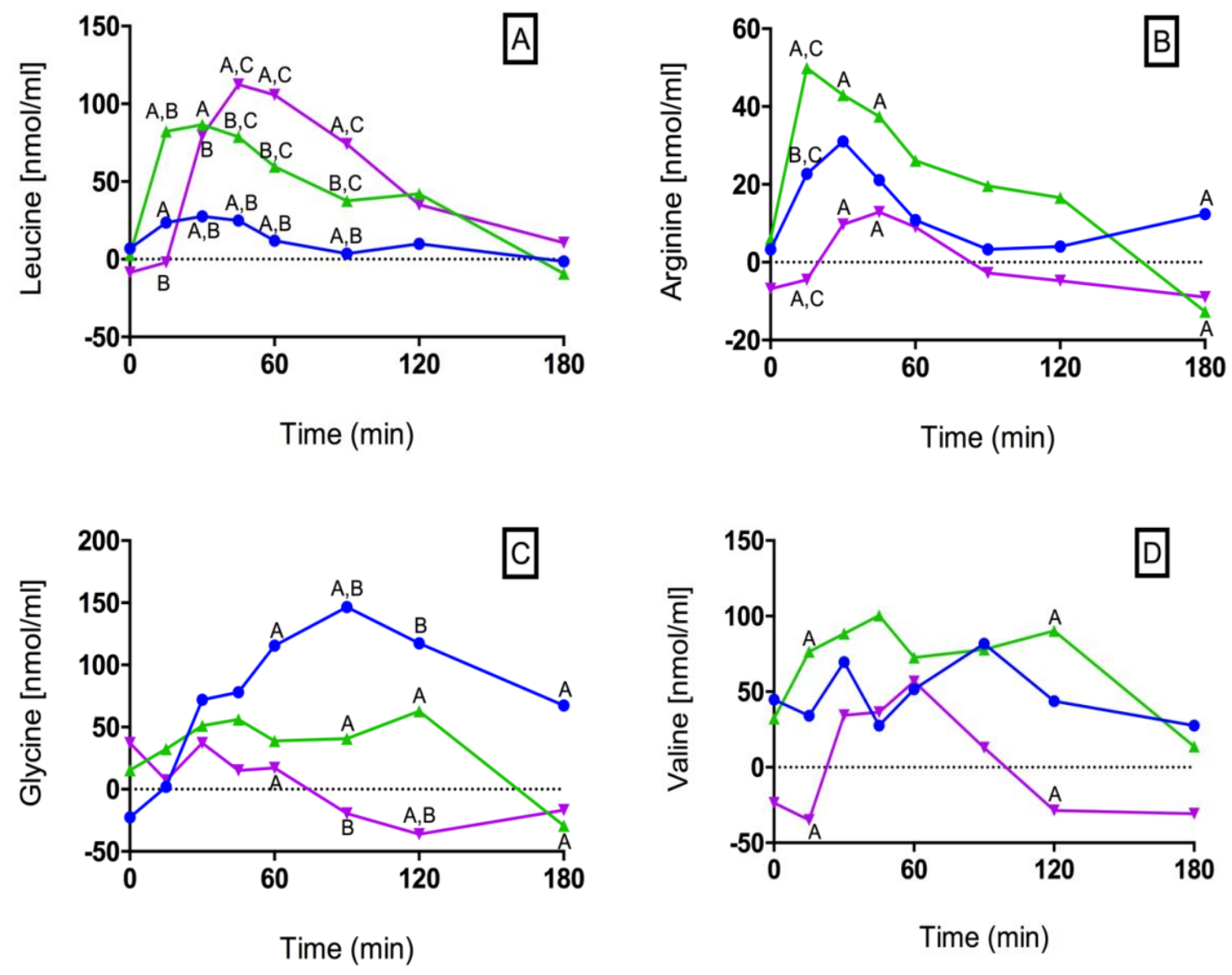

$\neq \mathrm{MyoCHX}{ }^{\mathrm{TM}} \rightarrow$ Beeflso $^{\mathrm{TM}} \rightarrow$ WPC

Figure 4. Plasma concentrations of individual amino acids. Mean plasma concentrations of leucine (panel A), arginine (panel B), glycine (panel C), and valine (panel D) following ingestion of WPC, BeefISO ${ }^{\mathrm{TM}}$, and MyoCHX ${ }^{\mathrm{TM}}$. Proteins sharing a common letter at a particular time point were found to have statistically different $(\mathrm{P} \leq 0.05)$ postprandial plasma amino acid responses.

\section{DISCUSSION}

Numerous protein sources are available to athletes and other adults who wish to build or retain muscle tissue and enhance recovery after rigorous exercise and training sessions. However, there is continuing debate about whether specific types of proteins (dairy, soy, meat, etc.) have a superior impact on MPS. Certain studies have shown that the source of protein is an important factor in determining the extent of MPS following protein ingestion $[4,12,13]$; potential factors could include differences in the amino acid composition or relative digestibility of the protein source. While much of the research on protein ingestion and MPS has been conducted using dairy, soy, or egg proteins, research studies also indicate that meat proteins are a key source of protein for stimulating muscle growth and improving strength gains [20]. The purpose of the present study was to evaluate the plasma amino acid response following post-exercise consumption of protein preparations from dairy (WPC), beef (BeefISO ${ }^{\mathrm{TM}}$ ), and chicken (MyoCHX ${ }^{\mathrm{TM}}$ ) sources. 
In an attempt to better characterize the composition of our test proteins, we compared the amino acid composition and peptide profile of WPC, a beef protein isolate (BeefISO ${ }^{\mathrm{TM}}$ ), and a hydrolyzed chicken protein isolate $\left(\mathrm{MyoCHX}^{\mathrm{TM}}\right)$. Amino acid composition analysis showed considerably higher levels of EAAs and BCAAs for both WPC and MyoCHX ${ }^{\mathrm{TM}}$ when compared to BeefISO ${ }^{\mathrm{TM}}$. Our results revealed in general the postprandial plasma amino acid response mirrored the amino acid composition for the protein sources. The amino acids found at the four highest levels for each of the protein sources were as follows (descending order): WPC glutamic acid/glutamine, aspartic acid, leucine, lysine; BeefISO ${ }^{\mathrm{TM}}$ - glycine, glutamic acid/glutamine, proline, alanine; MyoCHX ${ }^{\mathrm{TM}}$ - glutamic acid/glutamine, aspartic acid, lysine, leucine. There were also differences in the protein/peptide composition of each protein source. The majority of the protein content for both WPC and BeefISO ${ }^{\mathrm{TM}}$ consisted of peptides ranging from 5-15 kDa in size (approximately 36 to 107 amino acids long). MyoCHX ${ }^{\mathrm{TM}}$, on the other hand, is a hydrolysate of chicken protein which was confirmed by our results demonstrating that over $85 \%$ of the protein content consisted of free AAs or peptides smaller than $5 \mathrm{kDa}$. We included MyoCHX ${ }^{\mathrm{TM}}$ in our study for two reasons: hydrolyzed proteins are commonly used in sports nutrition products; and secondly, they are believed to provide a protein source that is more rapidly digested and absorbed, which improves postprandial plasma amino acid availability and may cause greater increases in MPS compared to intact protein [21, 22]. The hydrolyzed nature of the MyoCHX ${ }^{\mathrm{TM}}$ was further confirmed during our evaluation of postprandial plasma amino acid levels, where circulating levels of total AAs, EAAs, and BCAAs appeared much earlier compared to plasma amino acid levels in subjects consuming WPC or BeefISO ${ }^{\mathrm{TM}}$. Peak plasma amino acid levels typically appeared by 15-30 minutes when subjects consumed MyoCHX ${ }^{\mathrm{TM}}$ but not until after 30-60 minutes for WPC or BeefISO ${ }^{\mathrm{TM}}$. While postprandial plasma amino acid levels tended to increase and then decrease sharply after consuming WPC, the decline in postprandial amino acid levels was more prolonged after ingestion of MyoCHX ${ }^{\mathrm{TM}}$ or BeefISO ${ }^{\mathrm{TM}}$.

It is widely believed that essential amino acids (EAAs) are primarily responsible for stimulation of skeletal muscle protein synthesis, with little requirement for non-essential amino acids [23-25], an effect that can be further enhanced by prior resistance exercise [1, 3, 4]. Leucine in particular has been given special attention because of its unique secondary role as a nutrient signal to initiate muscle protein synthesis through the rapamycin (mTOR) signaling pathway $[9,10]$. This unique role for leucine in triggering translation initiation has led to the assumption that leucine content is the primary factor for consideration when defining quantity and quality of dietary proteins necessary to stimulate MPS [26]. However, results from a number of studies have cast doubt on this hypothesis by demonstrating a lack of enhancement in the rate of MPS by leucine infusion [27] or when leucine was added to an EAA solution or protein supplement $[28,29]$.

We found the amount of leucine in BeefISO ${ }^{\mathrm{TM}}$ is approximately $4 \%(\mathrm{w} / \mathrm{w})$, which some would argue is below the levels needed to stimulate adequate MPS. As one would expect, our results showed lower plasma leucine levels following intake of BeefISO ${ }^{\text {TM }}$ compared to WPC and $\mathrm{MyoCHX}^{\mathrm{TM}}$ (Figure 4). However, results from a performance study evaluating the effects of these proteins (46 grams of WPC, beef or chicken protein post-exercise) on body composition and muscle strength and power [30], showed similar improvements in lean body mass and fat mass and resistance training-induced strength adaptations among subjects in all test protein 
groups (WPC, BeefISO ${ }^{\mathrm{TM}}$, or MyoCHX ${ }^{\mathrm{TM}}$ ). This suggests that leucine, while important, is not solely responsible for activation of MPS and enhancement of body composition and strength, and that other factors (e.g. nonessential AA, proteins, and peptides) may also be involved. For example, plasma levels of valine (another EAA) following ingestion of BeefISO ${ }^{\mathrm{TM}}$ tended to be comparable to MyoCHX ${ }^{\mathrm{TM}}$ and higher than WPC (Figure 4), while consumption of BeefISO ${ }^{\mathrm{TM}}$ resulted in higher plasma glycine levels compared to WPC or MyoCHX ${ }^{\mathrm{TM}}$ (Figure 4). Our results also suggest that the earlier appearance of AAs in the blood stream following ingestion of hydrolyzed protein, as occurred with the MyoCHX $\mathrm{HM}^{\mathrm{TM}}$ group in our study, did not provide an advantage in terms of gains in body composition and strength performance [30].

Research has shown that both the initiation and the duration of postprandial stimulation of MPS in skeletal muscle may not be solely determined by the rise in blood leucine concentrations under rested or post-exercise conditions in young men [26, 31]. A study by Churchward-Venne, et al. [11] provided evidence to suggest intake of relatively low levels of leucine $(\sim 0.75 \mathrm{~g})$ may be sufficient to activate and induce a maximal stimulation of MPS in young healthy individuals, provided adequate amounts of the other EAAs (e.g. $\sim 8.5 \mathrm{gm}$ ) are also provided. Other reports have shown as little as $10 \mathrm{~g}$ of EAAs is sufficient to maximally stimulate MPS under both resting and post-exercise conditions in young healthy subjects [3, 32].

Alternatively, the gains in body composition and performance observed for BeefISO ${ }^{\mathrm{TM}}$ and MyoCHX ${ }^{\mathrm{TM}}$ might be explained by the role of other factors that may contribute to stimulating MPS, such as creatine in beef [33, 34] or other amino acids [35, 36]. For example, we found very high levels of glycine in BeefISO ${ }^{\mathrm{TM}}$ and higher plasma glycine levels following ingestion of BeefISO $^{\mathrm{TM}}$ compared to WPC or MyoCHX ${ }^{\mathrm{TM}}$ (Figure 4). Glycine may contribute to MPS and gains in body composition indirectly by supporting the synthesis and metabolism of creatine, which has been shown to improve lean body mass [37]. Creatine, along with phosphocreatine, plays a key role in cellular production and utilization of ATP [38], particularly in skeletal muscle. Because creatine is spontaneously converted to creatinine and excreted in urine, it must be continuously replaced by a combination of diet and endogenous synthesis. Creatine synthesis requires the whole glycine molecule, the amidino group of arginine, and methylation typically provided by methionine [38]. Due to consumption of the entire glycine molecule in creatine synthesis it has been estimated that $16 \%$ of daily dietary glycine intake is required for creatine synthesis alone [38]. Consequently, supporting endogenous creatine synthesis with increased dietary consumption of glycine may support muscle protein synthesis and begin to explain the improvements in lean body mass associated with resistance exercise and BeefISO ${ }^{\mathrm{TM}}$ supplementation.

Current nutritional paradigms rely solely on leucine content as a proxy for the potential of a protein to stimulate MPS. While leucine is widely accepted as a nutritional stimulus of mTOR and MPS, the variable impact on body composition observed with proteins having different EAA, BCAA, and total amino acid content underscores the need to consider nutritional supplements for their total potential benefit rather than as a delivery system for single nutrients (e.g. leucine). Potential benefits beyond amino acid composition that should be considered may include physiological benefits of native protein structures, functional peptides, energetics, and other characteristics that may extend the duration of protein synthesis. However, these potential added benefits of protein preparations beyond their amino acid contribution (e.g. peptide content, 
protein structure, etc.) must continue to be elucidated using precisely controlled clinical studies to more completely understand the benefits of functional foods beyond their strict nutritive value.

\section{CONCLUSIONS}

The nutritional composition, protein/peptide molecular weight, and postprandial plasma amino acid responses of WPC, BeefISO ${ }^{\mathrm{TM}}$, and $\mathrm{MyoCHX}^{\mathrm{TM}}$ were quantified to better understand the digestibility of these protein preparations. WPC was shown to have high EAA and BCAA content and led to maximum plasma amino acid concentrations within 30 minutes. While MyoCHX ${ }^{\mathrm{TM}}$ contained comparable levels of EAA to WPC, $76 \%$ of the protein comprising MyoCHX ${ }^{\mathrm{TM}}$ is in the form of small peptides $(<2 \mathrm{kDa})$, which led to accelerated peak plasma amino acid levels after $15 \mathrm{~min}$. BeefISO ${ }^{\mathrm{TM}}$ contained higher levels of CEAAs than the other two supplements and was the only protein source to exhibit a sustained release of amino acids that appeared in the blood and remained elevated over 3 hours. A recent clinical study compared post-workout supplementation with WPC, BeefISO ${ }^{\mathrm{TM}}$, or $\mathrm{MyoCHX}^{\mathrm{TM}}$ and showed similar improvements in lean body mass gains, loss of fat mass, and improvements in strength performance during an 8-week resistance-exercise training program. The similar post-workout performance of these protein supplements despite differences in amino acid content, peptide/protein molecular weight, and kinetics of the amino acid absorption, indicate the need to account for total nutritional potential when considering a protein supplement.

Abbreviations: BCAA, branched chain amino acids; CEAA, conditionally essential amino acids; EAA, essential amino acids; BeefISO, beef protein isolate; MyoCHX, hydrolyzed chicken protein hydrolysate; WPC, whey protein concentrate.

Competing interests: CJD, NJ, and EMW are employees of Essentia Protein Solutions. BWP is a consultant of Essentia Protein Solutions.

Author contributions: CJD, EMW were involved in study design, data collection and performing statistical analysis of the study data. BWP and CJD were involved in interpreting study data and writing the manuscript. All authors were involved providing critical review and approval of the final manuscript. The results provided in this manuscript do not constitute endorsement of the product by the authors.

Acknowledgements and funding: The authors would like to thank Dr. Jacob Wilson, Matthew Sharp, and Ryan Lowery of the Applied Science and Performance Institute for their involvement and review of the study design as well as conducting the study. This study was funded by Essentia Protein Solutions.

\section{REFERENCES}

1. Biolo, G., K.D. Tipton, S. Klein, and R.R. Wolfe, An abundant supply of amino acids enhances the metabolic effect of exercise on muscle protein. Am J Physiol, 1997. 273(1 Pt 1): p. E122-9.

2. Moore, D.R., S.M. Phillips, J.A. Babraj, K. Smith, and M.J. Rennie, Myofibrillar and collagen protein synthesis in human skeletal muscle in young men after maximal shortening and lengthening contractions. Am J Physiol Endocrinol Metab, 2005. 288(6): p. E1153-9. 
3. Moore, D.R., M.J. Robinson, J.L. Fry, J.E. Tang, E.I. Glover, S.B. Wilkinson, T. Prior, M.A. Tarnopolsky, and S.M. Phillips, Ingested protein dose response of muscle and albumin protein synthesis after resistance exercise in young men. Am J Clin Nutr, 2009. 89(1): p. 161-8.

4. Tang, J.E., D.R. Moore, G.W. Kujbida, M.A. Tarnopolsky, and S.M. Phillips, Ingestion of whey hydrolysate, casein, or soy protein isolate: effects on mixed muscle protein synthesis at rest and following resistance exercise in young men. J Appl Physiol (1985), 2009. 107(3): p. 987-92.

5. Drummond, M.J., H.C. Dreyer, B. Pennings, C.S. Fry, S. Dhanani, E.L. Dillon, M. Sheffield-Moore, E. Volpi, and B.B. Rasmussen, Skeletal muscle protein anabolic response to resistance exercise and essential amino acids is delayed with aging. J Appl Physiol (1985), 2008. 104(5): p. 1452-61.

6. Dideriksen, K.J., S. Reitelseder, S.G. Petersen, M. Hjort, I.C. Helmark, M. Kjaer, and L. Holm, Stimulation of muscle protein synthesis by whey and caseinate ingestion after resistance exercise in elderly individuals. Scand J Med Sci Sports, 2011. 21(6): p. e372-83.

7. Tang, J.E., J.J. Manolakos, G.W. Kujbida, P.J. Lysecki, D.R. Moore, and S.M. Phillips, Minimal whey protein with carbohydrate stimulates muscle protein synthesis following resistance exercise in trained young men. Appl Physiol Nutr Metab, 2007. 32(6): p. 1132-8.

8. Fujita, S., H.C. Dreyer, M.J. Drummond, E.L. Glynn, J.G. Cadenas, F. Yoshizawa, E. Volpi, and B.B. Rasmussen, Nutrient signalling in the regulation of human muscle protein synthesis. J Physiol, 2007. 582(Pt 2): p. 813-23.

9. Wullschleger, S., R. Loewith, and M.N. Hall, TOR signaling in growth and metabolism. Cell, 2006. 124(3): p. 471-84.

10. Suryawan, A., A.S. Jeyapalan, R.A. Orellana, F.A. Wilson, H.V. Nguyen, and T.A. Davis, Leucine stimulates protein synthesis in skeletal muscle of neonatal pigs by enhancing mTORCl activation. Am J Physiol Endocrinol Metab, 2008. 295(4): p. E868-75.

11. Churchward-Venne, T.A., N.A. Burd, C.J. Mitchell, D.W. West, A. Philp, G.R. Marcotte, S.K. Baker, K. Baar, and S.M. Phillips, Supplementation of a suboptimal protein dose with leucine or essential amino acids: effects on myofibrillar protein synthesis at rest and following resistance exercise in men. J Physiol, 2012. 590(Pt 11): p. 2751-65.

12. Pennings, B., Y. Boirie, J.M. Senden, A.P. Gijsen, H. Kuipers, and L.J. van Loon, Whey protein stimulates postprandial muscle protein accretion more effectively than do casein and casein hydrolysate in older men. Am J Clin Nutr, 2011. 93(5): p. 997-1005.

13. Wilkinson, S.B., M.A. Tarnopolsky, M.J. Macdonald, J.R. Macdonald, D. Armstrong, and S.M. Phillips, Consumption of fluid skim milk promotes greater muscle protein accretion after resistance exercise than does consumption of an isonitrogenous and isoenergetic soyprotein beverage. Am J Clin Nutr, 2007. 85(4): p. 1031-40.

14. Cotton, P.A., A.F. Subar, J.E. Friday, and A. Cook, Dietary sources of nutrients among US adults, 1994 to 1996. J Am Diet Assoc, 2004. 104(6): p. 921-30.

15. U.S. Department of Agriculture, A.R.S., Composition of Foods Raw, Processed, Prepared USDA Nutrient Database for Standard Reference, Release 15. . 2002.

16. Campbell, W.W., M.L. Barton, Jr., D. Cyr-Campbell, S.L. Davey, J.L. Beard, G. Parise, and W.J. Evans, Effects of an omnivorous diet compared with a lactoovovegetarian diet on 
resistance-training-induced changes in body composition and skeletal muscle in older men. The American journal of clinical nutrition, 1999. 70(6): p. 1032-9.

17. Hartman, J.W., J.E. Tang, S.B. Wilkinson, M.A. Tarnopolsky, R.L. Lawrence, A.V. Fullerton, and S.M. Phillips, Consumption of fat-free fluid milk after resistance exercise promotes greater lean mass accretion than does consumption of soy or carbohydrate in young, novice, male weightlifters. The American journal of clinical nutrition, 2007. 86(2): p. 373-81.

18. Karamać, M. and A. Rybarczyk, Chymotryptic hydrolysis of lentil meal proteins and characteristics of the resulting hydrolysates. Pol. J. Food Nutr. Sci., 2008. 58(3): p. 351357.

19. Badawy, A.A., The EZ:Faast family of amino acid analysis kits: application of the GC-FID kit for rapid determination of plasma tryptophan and other amino acids. Methods Mol Biol, 2012. 828: p. 153-64.

20. Robinson, M.J., N.A. Burd, L. Breen, T. Rerecich, Y. Yang, A.J. Hector, S.K. Baker, and S.M. Phillips, Dose-dependent responses of myofibrillar protein synthesis with beef ingestion are enhanced with resistance exercise in middle-aged men. Appl Physiol Nutr Metab, 2013. 38(2): p. 120-5.

21. Calbet, J.A. and J.J. Holst, Gastric emptying, gastric secretion and enterogastrone response after administration of milk proteins or their peptide hydrolysates in humans. Eur J Nutr, 2004. 43(3): p. 127-39.

22. Koopman, R., N. Crombach, A.P. Gijsen, S. Walrand, J. Fauquant, A.K. Kies, S. Lemosquet, W.H. Saris, Y. Boirie, and L.J. van Loon, Ingestion of a protein hydrolysate is accompanied by an accelerated in vivo digestion and absorption rate when compared with its intact protein. Am J Clin Nutr, 2009. 90(1): p. 106-15.

23. Borsheim, E., K.D. Tipton, S.E. Wolf, and R.R. Wolfe, Essential amino acids and muscle protein recovery from resistance exercise. Am J Physiol Endocrinol Metab, 2002. 283(4): p. E648-57.

24. Volpi, E., H. Kobayashi, M. Sheffield-Moore, B. Mittendorfer, and R.R. Wolfe, Essential amino acids are primarily responsible for the amino acid stimulation of muscle protein anabolism in healthy elderly adults. Am J Clin Nutr, 2003. 78(2): p. 250-8.

25. Tipton, K.D., B.E. Gurkin, S. Matin, and R.R. Wolfe, Nonessential amino acids are not necessary to stimulate net muscle protein synthesis in healthy volunteers. J Nutr Biochem, 1999. 10(2): p. 89-95.

26. Norton, L.E., D.K. Layman, P. Bunpo, T.G. Anthony, D.V. Brana, and P.J. Garlick, The leucine content of a complete meal directs peak activation but not duration of skeletal muscle protein synthesis and mammalian target of rapamycin signaling in rats. J Nutr, 2009. 139(6): p. 1103-9.

27. Nair, K.S., R.G. Schwartz, and S. Welle, Leucine as a regulator of whole body and skeletal muscle protein metabolism in humans. Am J Physiol, 1992. 263(5 Pt 1): p. E928-34.

28. Glynn, E.L., C.S. Fry, M.J. Drummond, K.L. Timmerman, S. Dhanani, E. Volpi, and B.B. Rasmussen, Excess leucine intake enhances muscle anabolic signaling but not net protein anabolism in young men and women. J Nutr, 2010. 140(11): p. 1970-6. 
29. Koopman, R., L.B. Verdijk, M. Beelen, M. Gorselink, A.N. Kruseman, A.J. Wagenmakers, H. Kuipers, and L.J. van Loon, Co-ingestion of leucine with protein does not further augment post-exercise muscle protein synthesis rates in elderly men. Br J Nutr, 2008. 99(3): p. 571-80.

30. Sharp, M.H., R.P. Lowery, K.A. Shields, J.R. Lane, J.M. Partl, D.W. Hayes, J.T. Rauch, C.A. Holmer, J.R. Minivich, J.M. Wilson, and E.O. De Souza, The Effects of Beef, Chicken, or Whey Protein Post-Workout on Body Composition and Muscle Performance. J Strength Cond Research, 2016. (Accepted).

31. Rennie, M.J., J. Bohe, K. Smith, H. Wackerhage, and P. Greenhaff, Branched-chain amino acids as fuels and anabolic signals in human muscle. J Nutr, 2006. 136(1 Suppl): p. 264S$8 \mathrm{~S}$.

32. Cuthbertson, D., K. Smith, J. Babraj, G. Leese, T. Waddell, P. Atherton, H. Wackerhage, P.M. Taylor, and M.J. Rennie, Anabolic signaling deficits underlie amino acid resistance of wasting, aging muscle. FASEB J, 2005. 19(3): p. 422-4.

33. Volek, J.S., W.J. Kraemer, J.A. Bush, M. Boetes, T. Incledon, K.L. Clark, and J.M. Lynch, Creatine supplementation enhances muscular performance during high-intensity resistance exercise. J Am Diet Assoc, 1997. 97(7): p. 765-70.

34. Bird, S.P., Creatine supplementation and exercise performance: a brief review. J Sports Sci Med, 2003. 2(4): p. 123-32.

35. Atherton, P.J., K. Smith, T. Etheridge, D. Rankin, and M.J. Rennie, Distinct anabolic signalling responses to amino acids in C2C12 skeletal muscle cells. Amino Acids, 2010. 38(5): p. 1533-9.

36. Smith, K., N. Reynolds, S. Downie, A. Patel, and M.J. Rennie, Effects of flooding amino acids on incorporation of labeled amino acids into human muscle protein. Am J Physiol, 1998. 275(1 Pt 1): p. E73-8.

37. Branch, J.D., Effect of creatine supplementation on body composition and performance: $a$ meta-analysis. Int J Sport Nutr Exerc Metab, 2003. 13(2): p. 198-226.

38. Brosnan, J.T. and M.E. Brosnan, Creatine: endogenous metabolite, dietary, and therapeutic supplement. Annu Rev Nutr, 2007. 27: p. 241-61. 